\title{
Collective Phenomena in Heavy Ion Collisions
}

\author{
Slavica Brkić ${ }^{1, *}$, Mile Dželalijaª \\ ${ }^{1}$ Faculty of Natural Sciences, Mathematics and Education, University of Mostar, Bosnia and Herzegovina \\ ${ }^{2}$ Faculty of Natural Sciences and Mathematics, University of Split, Croatia
}

Copyright@2017 by authors, all rights reserved. Authors agree that this article remains permanently open access under the terms of the Creative Commons Attribution License 4.0 International License

\begin{abstract}
To create conditions which ruled one billionth of a second after the Big Bang, it is necessary to heat and compact the nuclear matter. During the first microseconds after the Big Bang the universe went through such a phase transition at very high temperatures but very low net baryon density. At very high temperatures or densities, the hadrons melt and their constituents, the quarks and gluons, form a new phase of matter, the so called quark-gluon plasma. Relativistic heavy ion collisions aim to create a quark gluon plasma where quarks and gluons can move freely over volumes that are large in comparison to the typical size of a hadron. When the particles collide at high energies, it leads to the conversion of particle collision participants in a much heavier particle. If the energy density is large enough, after a collision occurs the formation of quark-gluon plasma. In the dense nuclear medium, it comes to collective phenomena such as increased production of strangeness, damping charmonium and collective motion of particles. In nuclear medium, it comes to individual collision of quarks, which also hadronize. Using simulation package Pythia, we analyzed the reaction system that results in individual collisions of quarks and antiquarks, and emergence of collective phenomena.
\end{abstract}

Keywords Quark-gluon Plasma, Strange Particle, Charmonium, Collective Motion, Elliptic Flow

\section{Introduction}

The aim of the research programs in the new accelerators is the study of nuclear matter under extreme conditions. Extreme conditions include high temperature and low density or low temperature and high density. Nuclear matter as well as water can be found in the different phases as a function of temperature and density. Phase diagram of strongly interacting matter can be displayed by changing the energy.

The phase diagram has been more researched in the field of low density and high temperature than in the high densities and low temperatures. In future research in CERN
$\mathrm{LHC}^{1}$ the exploration of systems at higher temperatures and lower baryonic densities will be continued. These conditions existed in the early Universe when it suffered a phase transition from a quark-gluon plasma to hadronic matter in the first microseconds after the Big Bang [1]. To about three times larger than the core, Baryonic density is achieved in heavy-ion collisions (HIC) in SIS18 ${ }^{2}$ accelerator $\mathrm{GSI}^{3}$. The highest baryonic density is expected for nuclear collisions in the energy range of 10 to $40 \mathrm{GeV} / \mathrm{u}$ [2]. The matter in this form exists in neutron stars in the center of the supernova explosion. In the laboratory, the super dense nuclear matter can be formed by reaction of the collisions of large amounts of relativistic heavy ions. Much of the energy beam is converted to the excited baryonic resonance, which decays into pions and nucleons. This mixture of nucleons, baryonic resonance and mesons is called hadronic matter. At very high temperatures and/or densities hadrons melt and their constituents, quarks and gluons form a new phase of matter to quark-gluon plasma. The phase diagram of strongly interacting matter is shown in Figure 1[3]. "Liquid" phase is realized in the nucleus at zero temperature and density of 300million tons $/ \mathrm{cm}^{3}$. At low densities nucleons behave as a gas [1].

Different phases of heavy-ion collisions (HIC) are shown in Figure 2[4]. Two energy cores come along the light cone and collide with each other and create multipartonic systems. In the initial collisions, large amount of kinetic energy of colliding nuclei is used to create a large number of secondary particles in a small volume. These particles collide often enough to achieve a state of thermal equilibrium and the quark-gluon plasma (QGP) can be formed. In nuclear medium it comes to individual collision of quarks, which also hadronize. This is a transitional state: after the further expansion and cooling system again hadronize. The system is still expanding and cooling, leading to freeze-out[4]. First occurs chemical freeze-out; chemical freeze-out point is the state in the evolution of the hadronic system when inelastic collisions cease and relativities particles become fixed and

1 CERN LHC -Centre Europeene pour la Recherdhe Nuclearre-Large Hadron Collider (Geneve)

2 SIS-Silicon Integrated Systems (Darmstadt-Germany)

3 GSI-Gesellschaft fur Schwerionenforschung (Darmstadt-Germany) 
defined by temperature and the chemical potential $\mu_{B}$ [5]. Chemical freeze-out parameters at different energies collisions are grouped in a single line in $T-\mu_{B}$ plane. This line is called a freeze-out line and can be determined by average energy per particle $\langle E\rangle /\langle N\rangle \sim 1 \mathrm{GeV}$ [6]. After chemical freeze-out, the composition of the particles inside the fireball is fixed, but elastic collisions still hold the system together, to the final kinetic freeze-out where the shape of the transverse distribution of momentum is fixed. The spectrum of particles from that moment is seen with detector [7].

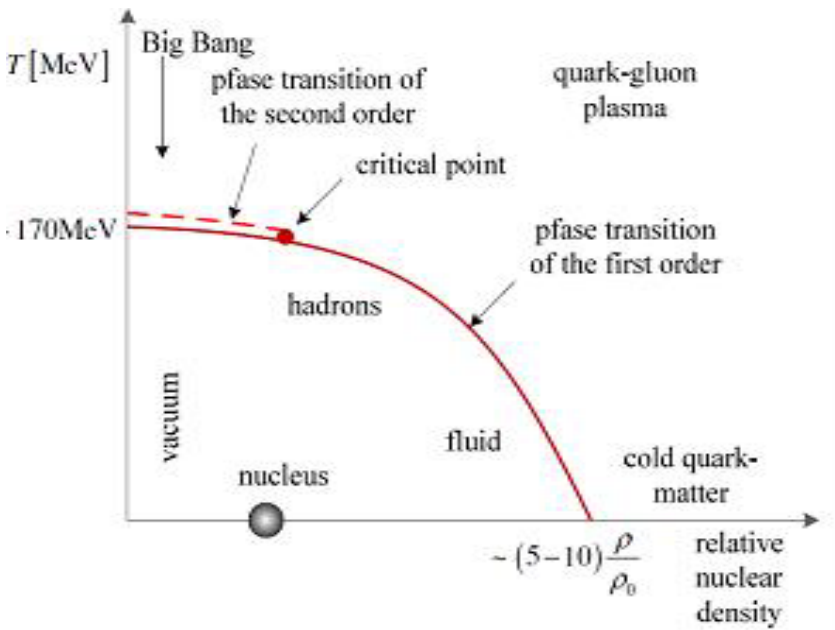

Figure 1. Phase diagram of nuclear matter

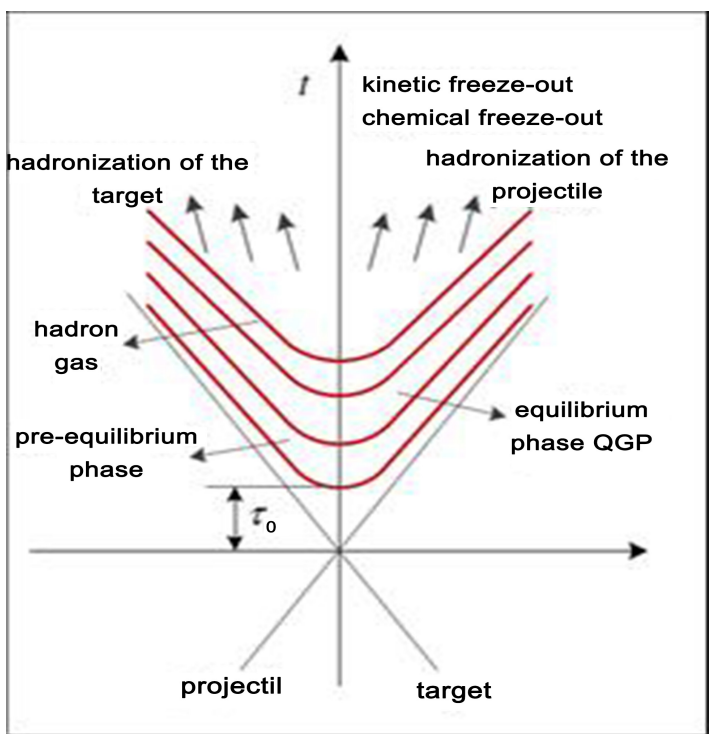

Figure 2. The dynamics of the collision

\section{Theoretical Background}

The heavy-ion collision experiments measure observables which are sensitive to effects of high baryon densities and to phase transitions. For example, they focus on the investigation of charmonium production, production of strange particles and collective flow of all observed particles.

\subsection{Enhanced Production of Strangeness}

In the collision of ions, original ions contain only up and down quarks; no strange quarks. The particles formed in a collision contain new up and down quarks and strange quarks. For electron-positron, proton-proton collisions part of strange quarks is 0.2 . The ratio is the same no matter how much the energy is increased. For nucleus-nucleus collisions part is twice as large, i.e., overall strangeness is increased twice. Increased production of strange particles was proposed as an experimental signal occurrence of quark-gluon plasma in central collisions of heavy nuclei.

Figure 3 shows the relation $K^{+} / \pi^{+}$as a function of beam energy for central $\mathrm{Au}-\mathrm{Au}$ collision, $\mathrm{Pb}-\mathrm{Pb}$ i p-p collision in the system of the center of mass [1]. Ratio of $\mathrm{K}+$ and $\pi^{+}$ meson yields as function of beam energy for central $\mathrm{Au}+\mathrm{Au}(\mathrm{Pb}+\mathrm{Pb})$ collisions in comparatio to data from p-p collisions (open symbols). The data were measured by the collaborations E899/E917[8] at the AGS and by NA49 at

CERN-SPS [9]. $K^{+} / \pi^{+}$relationship has a maximum between the top $\mathrm{AGS}^{4}(10.7 \mathrm{GeV} / \mathrm{u})$ and the lowest energy $\operatorname{SPS}^{5}(40 \mathrm{GeV} / \mathrm{u})$ in the laboratory system. The subject of debate is whether this nonmonotonic behavior is caused by phase transition or not. Statistical model description of difficult ion collisions provides that the contribution of strange compared to the non-strange particles exhibits a maximum around the beam energies around $30 \mathrm{GeV} / \mathrm{u}$. This increase was not observed in proton-proton collisions, which indicates that collective effects dominate in the production of strangeness in this energetic field. Increased production of strangeness in the collision of protons and ions is still an open question, whether it is the result of superposition compared to proton - proton or is caused by some other mechanism. The main arguments to explain the formation of the QGP in increasing strangeness is based on predictions that the threshold energy production $s \bar{s}$ pairs in the QGP is lower than in the hadronic gas and also that the production of pairs of strange quarks is preferred to light quarks [2]. 


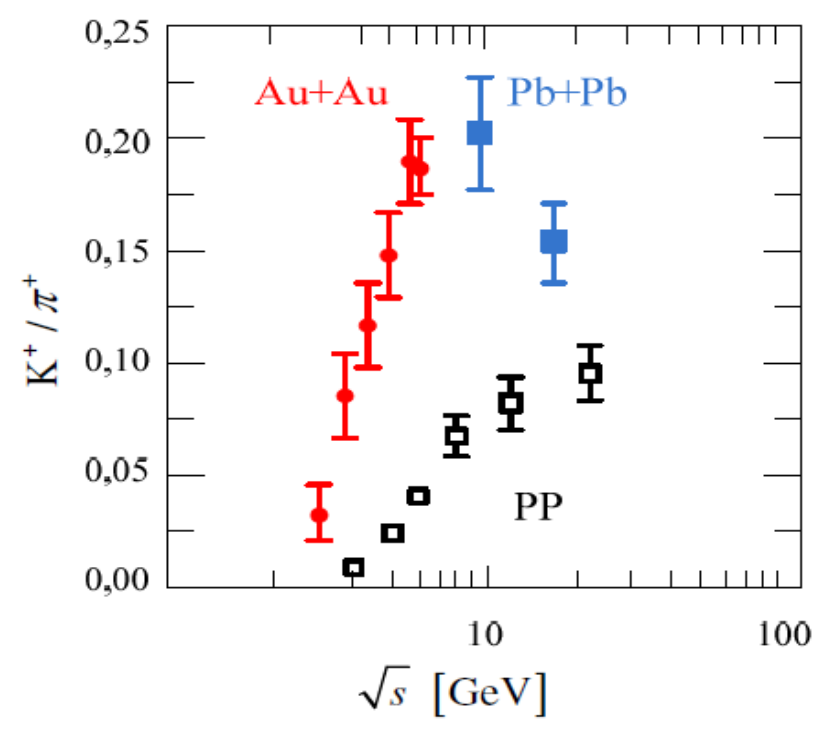

Figure 3. Energy distribution $K^{+} / \pi^{+}$in HIC and pp collision

\subsection{Suppression of Charmonium}

QGP signals associated with the production of $J / \Psi$ mesons. $J / \Psi$ particles are made of charm quark and anti-quark $c \bar{C}$. They are rare because charm quarks are very massive, and can be produced at an early state immediately after the collision, while the constituents of the nucleus still have full energy [10].

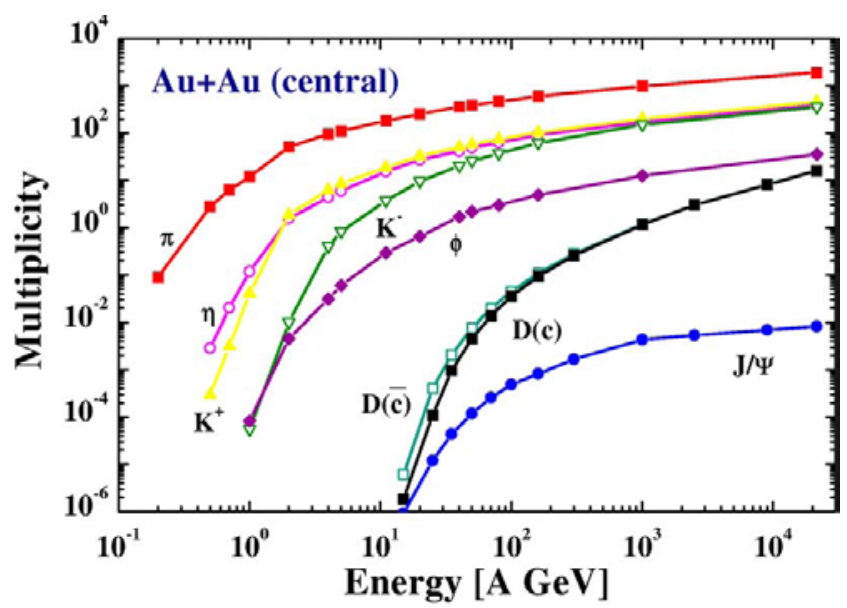

Figure 4. Energy dependent mesons multiplicity in $\mathrm{Au}-\mathrm{Au}$ central collision

However the formation of $J / \Psi$ mesons can be suppressed by the presence of QGP. That strong reduction in the number $J / \Psi$ leaving fireball suggests that the hot QGP is formed in the initial state of the collision [11]. Reduced production of charmonium is predicted decades ago. Matsui and Satz proposed in 1986. Attenuation of heavy mesons as a result of shading in the quark-gluon plasma; shading reduces the attractive forces between heavy quarks and antiquarks, which prevents the binding of charm quarks and antiquarks. Above a certain critical point shading prevents the formation of bound states. Suppression of $J / \Psi$ requires temperatures that are $30 \%$ above the color deconfinement temperature and energy density of about $3 \mathrm{GeV} / \mathrm{fm}^{3}$. This agrees with the initial energy densities achieved in $\mathrm{Pb}-\mathrm{Pb}$ collisions [12]. Multiplicities of mesons produced in central $\mathrm{Au}+\mathrm{Au}$ collisions as function of beam energy according to the HSD transport code [13] is presented in Figure 4.

\subsection{Collective Flow}

The collective flow of particles was theoretically predicted to occur in relativistic heavy-ion collisions already in the early seventies of the last century [14]. They were provided for two signature flows: a) Deflection of compressed matter in the reaction plane, deflection to the side, spectator fragments - "bounce off", known as a directed flow of nucleons overlapping region of colliding nuclei in the reaction plane [15], b) leakage ("squeeze-out") of participants outside the reaction plane, called elliptic flow [16]. It was first experimentally detected in the nucleus-nucleus collision Plastic Ball collaboration of the National Berkley Laboratory (NBL) in 1984. For charged particles [17] but may be present in the proton - proton collisions [18]. Various phenomena of the flow are observed at energies between $50 \mathrm{MeV} / \mathrm{u}$ and $160 \mathrm{MeV} / \mathrm{u}$. Starting spatial anisotropy gives rise to final anisotropy of momentum, on account of multiple between particle collisions (Figure 5 and 6)[19]. If one of the two factors, called the initial anisotropy and scattering, is omitted, it is not the flow. Thus in the non-central collisions, the flow enables measurement of scattering. In other words, the flow is sensitive to the number of interactions and cross section scattering parton-parton [20].

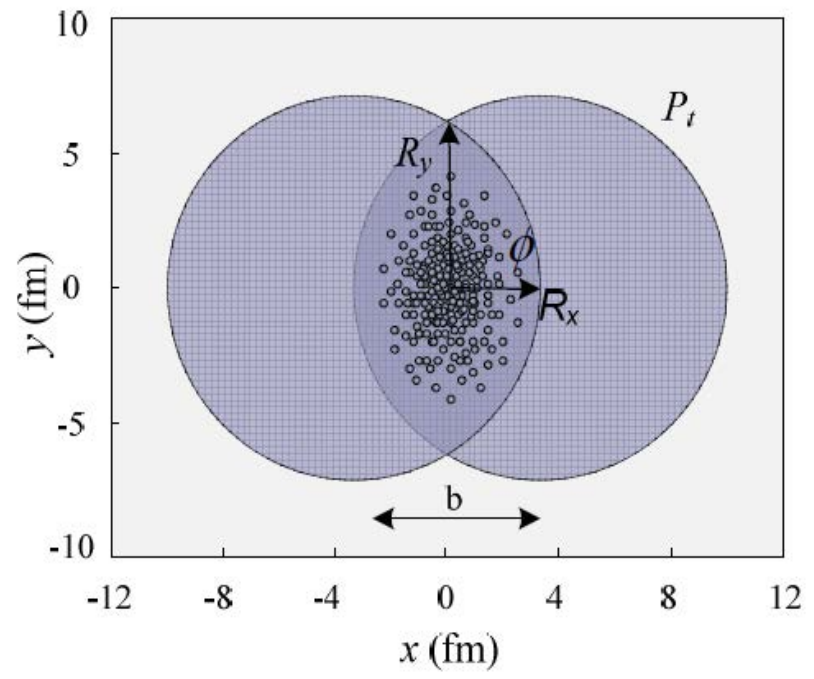

Figure 5. Overlap region of projectile and target 


\subsubsection{Transversal Flow}

The transversal flow is a collective movement in transversal level. In central collisions between the spherical nucleus of transverse pressure gradients are independent of the azimuthal corner and field of transverse flow is azimuthally isotropic. In non-central collisions between the spherical core, or central collisions between deformed nuclei $(U+U)$, nuclear overlapping region is initially spatially deformed in the transverse plane, resulting in the azimuthal anisotropy of the pressure gradient and the final shape of the source.

Anisotropic flow is defined as the different $n$-th harmonic coefficient $v_{n}$ of the Fourier expansion for the particle invariant azimutal distribution [21]

$$
\frac{d N}{d \varnothing} \propto 1+2 \sum_{n=1}^{\infty} v_{n} \cos (n \varnothing)
$$

where $\varnothing$ is the azimutal angle between the transverse momentum of the particle and the reaction plane. Note that the $\mathrm{z}$-axis is defined as the direction along the beam and the impact parameter axis which is labeled as X-axis. Anisotropic flows generally depend on both particle transverse momentum and rapidity, and for a given rapidity the anisotropic flows at transverse momentum $p_{t}=$ $\sqrt{p_{x}^{2}+p_{y}^{2}}$ can be evaluated accoding to

$$
v_{n}\left(p_{t}\right)=\langle\cos (n \emptyset)\rangle
$$

where $\langle\cos (n \emptyset)\rangle$ denotes average over the azimutal distribution of particles with transverse momentum $p_{t}$.

Directed flow is described with the first Fourier harmonic [22]:

$$
v_{1}=\langle\cos \varphi\rangle=\left\langle\frac{p_{x}}{p_{t}}\right\rangle
$$

Elliptic flow is described with the second Fourier coefficient [20] (Figure 7):

$$
v_{2}=\langle\cos 2 \phi\rangle=\left\langle\left(\frac{p_{x}}{p_{t}}\right)^{2}-\left(\frac{p_{y}}{p_{t}}\right)^{2}\right\rangle
$$

Two specific forms of anisotropy of the transverse flow fields are "directed" and "elliptic" flows. The word "directed" comes from the fact that such a course has a direction, and the word "elliptic" is due to the fact that in polar coordinates azimuth distribution with nonzero second harmonic represents an ellipse. In the one-fluid hydrodynamics, the concept of the transverse flow is particularly simple. Pressure gradient between the dense center of the system and the vacuum causes the expansion of the system and the transverse velocity of the fluid increases. But if you define the size of the system with the local area freeze, the speed of the transverse flow is not the expansion rate of the system. As the system can be thinned, surface freeze-out can be moved inward, even if particles of fluid are flowing quickly to the outside [7].

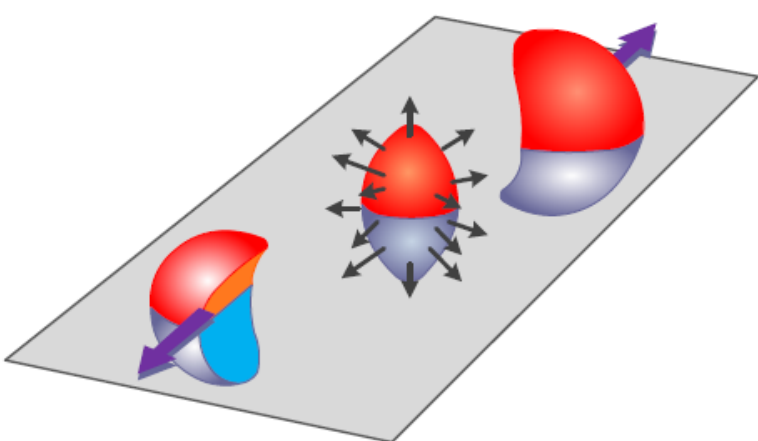

initial spatialanisotropy
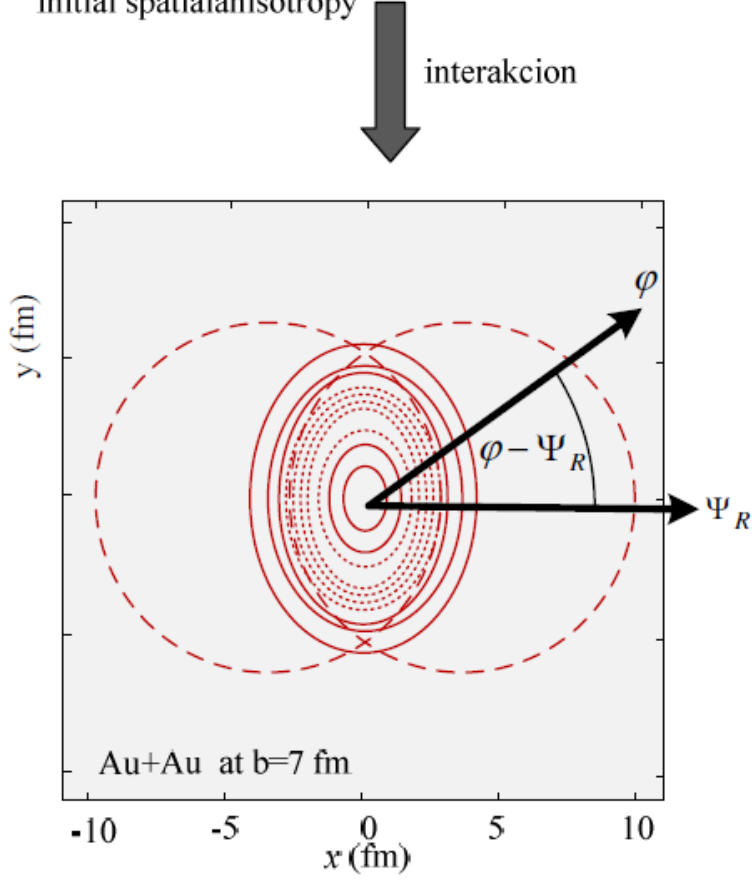

final anisotropy of momentum

Figure 6. Initial spatial anisotropy and final anisotropy of momentum

\subsubsection{Elliptic Flow}

In non-central collisions production of primary particles is azimuthally isotropic, but as the interaction area is anisotropic in space, secondary collisions can cause the distribution of final particles is anisotropic in space impulses. As shown in Figure 5, the particles that out of plane have a longer average distance of movement within a compact area from the particles moving in the plane. Thus particles moving out of plane are more likely to turn several times and change their direction than the particles moving in plane. Therefore the final distribution of the particles has more particles moving in-plane than out of plane and Fourier coefficient is positive [18]. Large elliptical anisotropy and large parameter value of elliptical flow is a sign of abundance scattering at an early stage of collision and therefore signal of the early lifting of pressure and thermalization. When the phase transitions collapse of the flow is predicted, the study of the flow gives data for the 
phase transitions.

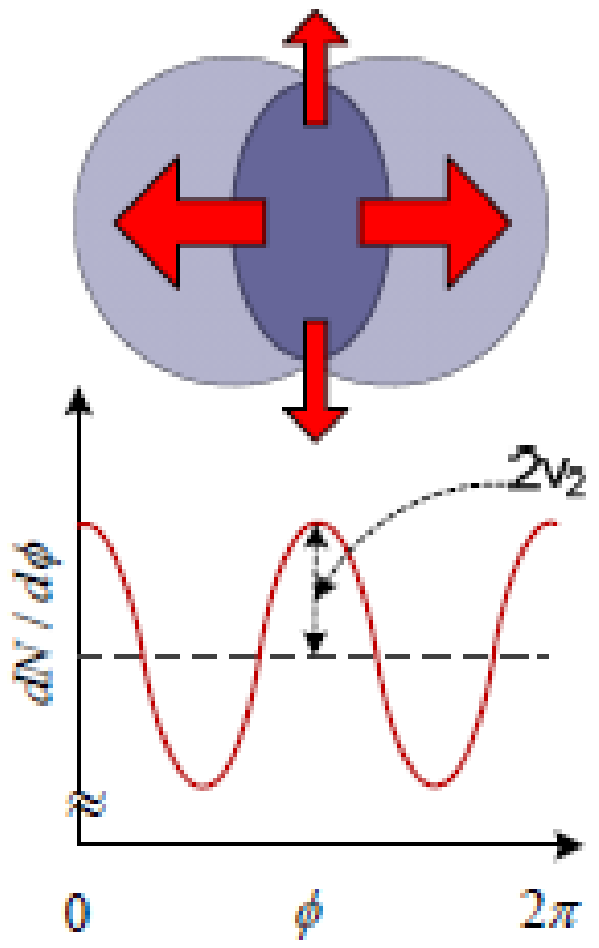

Figure 7. Anisotropic distribution of particle

Figure 8 shows typical changes of elliptic flow by collision energy[22]. Size of directed flow depends on the centrality of the collision. Vanishes for central collisions with symmetry and for very peripheral collisions where the compression effects are weak. Therefore the maximum of directed flow is for a semi-central collision, the impact parameter from 5-6fm for Au-Au collisions [20, 21]. The dependence of the collision parameter of elliptic flow is similar to that from of directed flow. However, the maximum is at a slightly higher impact parameter, about $7 \mathrm{fm}$ for $\mathrm{Au}-\mathrm{Au}$ collisions.

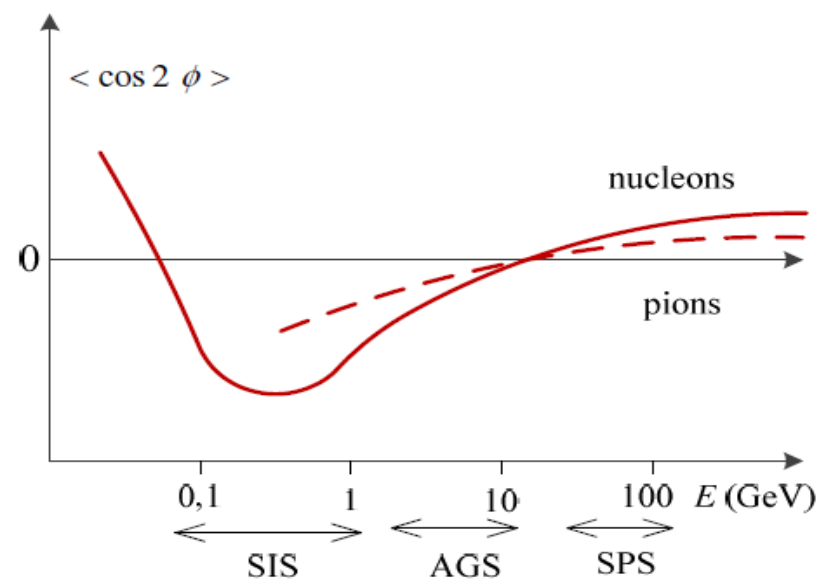

Figure 8. Energy dependence of elliptic flow

\section{Generating Events: Simulation of Collision of Individual Quarks and Antiquarks}

Using the Pythia simulation package we have collided quarks and antiquarks in relativistic and ultra-relativistic energy. In individual collisions the energy of quarks is complited transformed in heavier particles. The multiplicity and type of those particles is dependent of collision particles and collision energy as well. We have analyzed mentioned phenomena in individual collisions light and heavy quarks and antiquarks.

The production of strange particles. Enhanced production of strangeness has been proposed as a signal for the transient existence of a deconfined phase of quarks and gluons in nucleus-nucleus collisions at ultrarelativistic energies. The idea is that the production of strange quark pairs is energetically favored in the quark-gluon plasma as compared to hadronic matter. The simulation of collision up quark and up antiquark is shown in Figure 9.

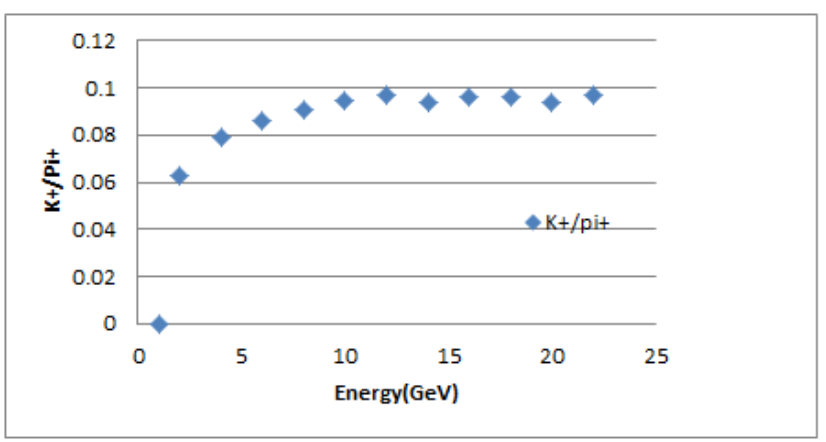

Figure 9. Energy distribution $\mathrm{K}^{+} / \pi^{+}$in u- quark u-antiquark collision for relativistic energy

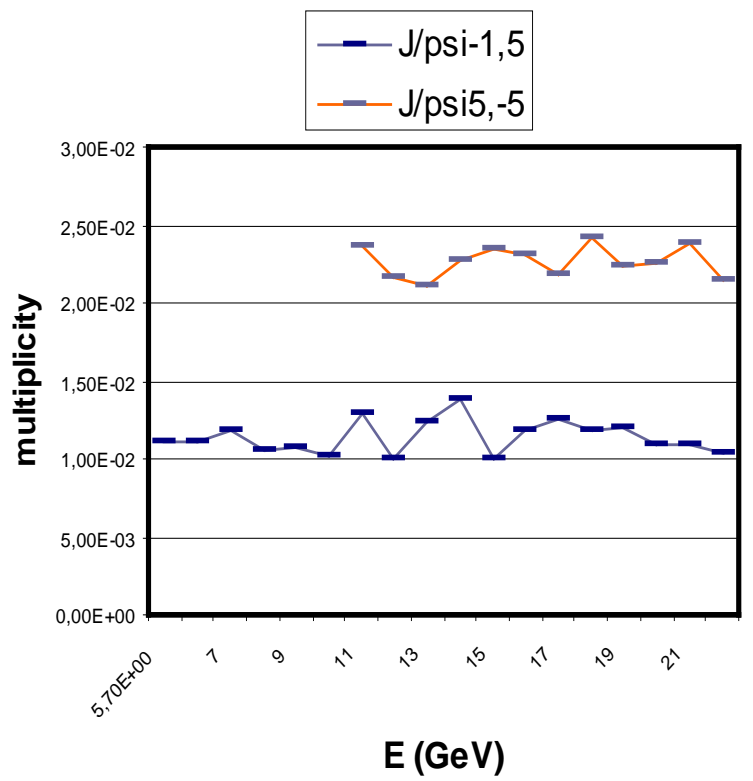

Figure 10. Energy dependent $J / \psi$ multiplicity in d-antiquark/b-quark $(-1,5)$ and b-quark/b-antiquark $(5,-5)$ collision

The production of $\mathbf{J} / \boldsymbol{\Psi}$ mesons. QGP signals associated 
with the production of $J / \Psi$ mesons. The production and propagation of charm in heavy ion collisions is expected to be a particularly sensitive probe of the hot and dense medium. The suppression of charmonium is predicted as a signature for the quark-gluon plasma [1]

In the collision of two light quarks, such as d-quark and d-antiquark to production $J / \Psi$ mesons doesn't come no matter how much we increase energy. In the collisions of heavy and light quarks (u-antiquark and b-quarks) comes to the production of $J / \Psi$ mesons with a threshold energy that is lower than in the collision of heavy quarks (Figure 10). Multiplicity of mesons produced is higher in the collision of heavy compared to the heavy and light quark.

Collective Flow. Because the collective flow has been observed in proton-proton collisions, we investigated the same occurrence of flow in quark-antiquark collisions. In the collision of individual quarks we have measured elliptic flow. The results of simulation are shown in Figure 11 and 12.

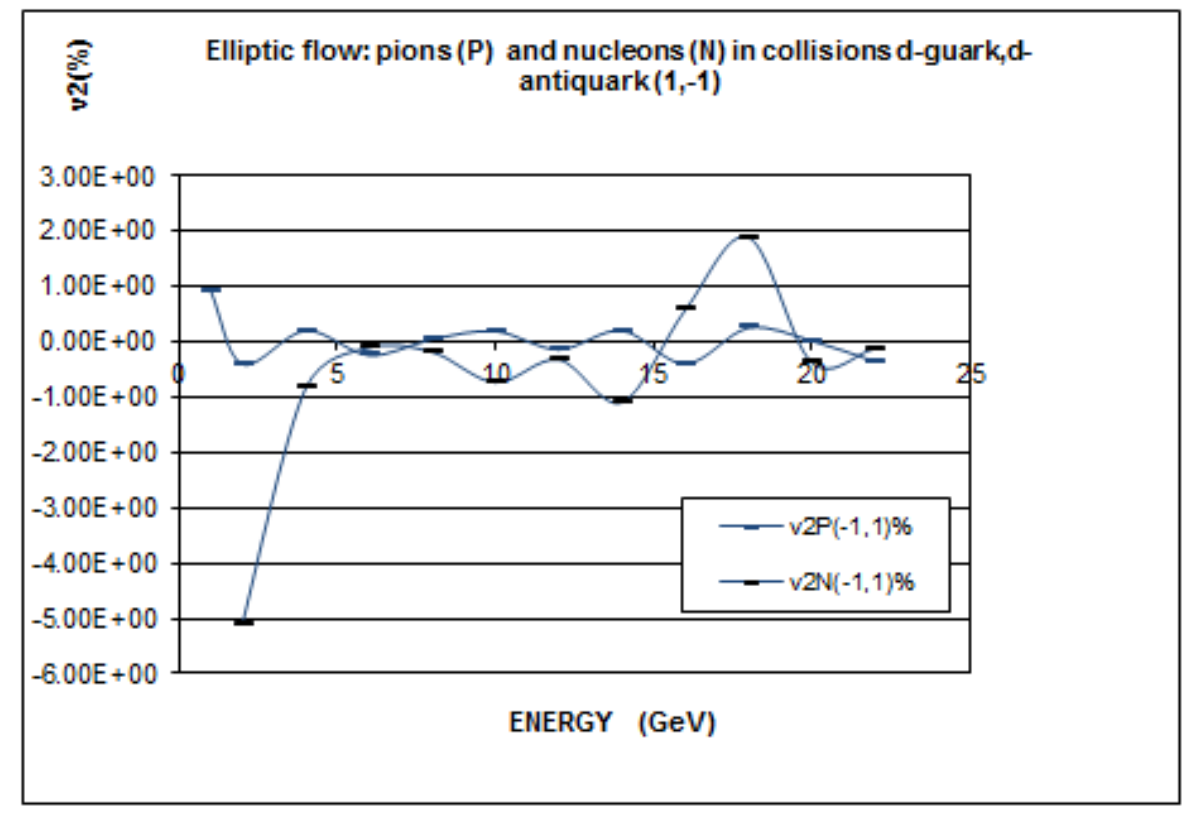

Figure 11. Energy dependence eliptic flow in collision d-quark/d-antiquark

Elliptic flow pions and nucleons in colliion d-antiquark/b-quark

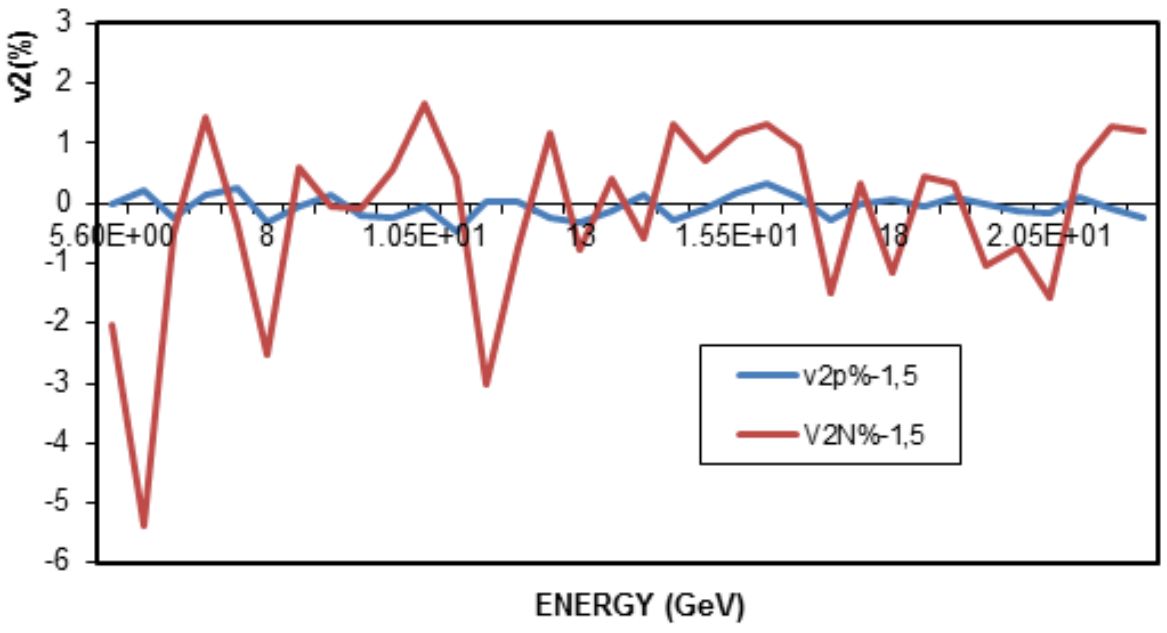

Figure 12. Energy dependence elliptic flow in collision d-antiquark /b-quark 


\section{Analysis of Results and Discussion}

The results of our simulations we compared with experimental and theoretical data related to the collisions of heavy ions performed in the well-known colliders such as AGS, SPS or NBL. In heavy ions collisions the hot and dense matter is created in which quarks move freely. Also, the short-lived existence of quark-gluon plasma could be related to the collective phenomena peculiar only for extreme conditions. In hot and dense matter the individual collisions of quarks occur and they start to transform into heavier particles. Because such individual collisions cannot be followed experimentally, only the results of simulations are used to describe the system behavior.

\subsection{Production of Strange Particles in Quark-antiquark Collisions}

In individual collisions of quarks there is no increased production of strangeness no matter how much we increase the energy. In the range of relativistic energy ratio of the strange and the non-strange particles is slightly below 0,1 (Figure 9) and for ultra-relativistic range that value is slightly above 0,1 (Figure 13). The ratio of strange and non-strange particles is around 0.1 as in case of proton-proton collisions (Figure 3)[7]. Value of ratio 0,1 appears in all cases regardless what kind of quarks and antiquark take part in the collisions.

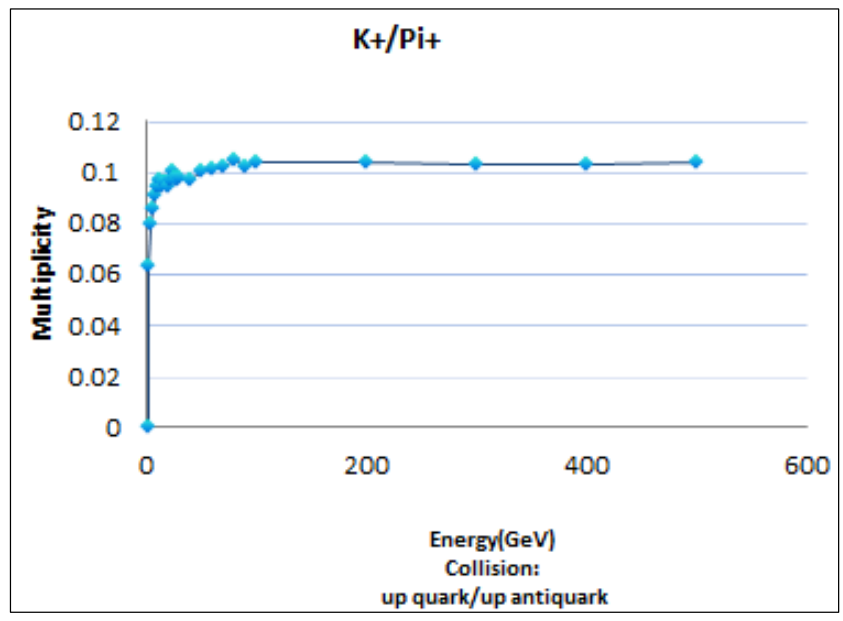

Figure 13. Energy distribution $K^{+} / \pi^{+}$in $u U$ - quark $u$-antiquark collision for relativistic and ultrarelativistic energy

\subsection{Production of $J / \Psi$ meson in Quark-antiquark Collisions}

An important predicted signature of free quarks involves a particle called the $J / \Psi$. In the collision of two light quarks, such as d-quark and d-antiquark to production $J / \Psi$ mesons doesn't come no matter how much we increase energy. In the collisions of heavy and light quarks (u-antiquark and b-quarks) comes to the production of $J / \Psi$ mesons with a threshold energy that is lower than in the collision of heavy quarks (Figure 10). Multiplicity of mesons produced is higher in the collision of heavy compared to the heavy and light quark.

The analysis of our results and a comparison with the production of $J / \Psi$ mesons in collisions of heavy ions (Figure 4) can be observed that the multiplicity is smaller in collisions of heavy ions than in the collision of quarks. The production of $J / \Psi$ mesons isn't suppressing as in the heavy ion collisions.

\subsection{What's Elliptic Flow in qq Collision}

We analyzed the energy dependence of elliptic flow for the reaction system created as a result of high-energy collisions of individual quarks. In collisions of quarks as well as in collisions of ions is the largest multiplicity of pions and of nucleons because they are made of light $\mathrm{u}$ - and d-quarks. We observed energy dependence of elliptical flow just for pions and nucleons as has been done for heavy ions. Figure 11 shows the energy dependence of elliptical flow at different energies in unsymmetrical collision of two light quarks and figure 12 show elliptic flow unsymmetrical collision of d-antiquark and b-quark. The distributions of pions and nucleons in the figures 11 and 12 are not a flow. Energy dependence has no typical form, which indicates that in the collision of heavy ions are formed collective phenomena that are not a superposition of individual reaction system.

\section{Conclusions}

Super dense nuclear matter can be created in a laboratory in collisions of relativistic heavy ions. If the energy density is large enough it leads to the formation of quark-gluon plasma. In the dense substances appears unusual behavior such as increased production of strangeness, suppression of charmonium or collective motion of particles. Increased production of strangeness, suppression of charm particles and collective motion are collective phenomena. They can be associated with transitory existence of quark-gluon plasma. We analyzed the reaction system resulting in a collision of individual quarks and antiquarks and showed that these phenomena do not occur. Ion-ion collisions are clearly distinct from nucleon-nucleon and quark-antiquark collisions. Collective behaviour of matter is occurring only under extreme conditions. The first observations show that the energy dependence of the elliptic flow for pions and nucleons is similar for collisions of gluons as it is for collisions of heavy-ions.

For the comprehensive and detailed conclusions, there is a need for further analysis of collective motions simulated for different elementary particles and conditions and comparing them to observations of heavy ion reactions, which now emerges in many experiments. 


\section{REFERENCES}

[1] P.Senger: The nucleus-nucleus collision research program, 2002.

[2] P.Senger: The Compressed Baryonic Matter Experiment, GSI, Darmstadt

[3] Shematic phase diagram for nuclear matter, Image file 96803723 LBNL,phasediagram 6.jpg, gsi.de

[4] T.Hirano, N van der Kolk, A. Bilandzic: arXiv:0808.2684, 2008.

[5] O.Ristea:Study of the chemical freeze-out in nucleus-nucleus collisions for BRAHMS Coll, 2004.

[6] J.Cleymans and K.Redlich, Phys. Rev. Lett. 81,5284 (1998)

[7] P.Huovinen:arXiv: nucl-th/0305064v1 2003.

[8] L. Ahle et al., Phys.Lett. B 490 (2000) 53

[9] J. Bächler et al., Nucl. Phys. A 661 (1999) 45c and H. Ströbele, priv. com.

[10] Nucleus-nucleus www.gsi.de/GSI-future/cr/PDF/
[11] A New State Matter created at CERN http://newstate-matter.web.cern.ch/

[12] S.A.Bass et al.: arXiv-hep/ph/9810281 v2 1998.

[13] W. Cassing, E. Bratkovskaya, A. Sibirtsev, Nucl. Phys. A 691 (2001) 74 j-psi

[14] H.Schied et al,//Phys. Rev. Lett.1968.V.21.P.1479. exp

[15] Stocker H. Et al.//Phys. Rev. Lett.1980.V. 44.P.725.

[16] Stocker H. Et al.//Phys. Rev. Lett.1984.V. 52.P.1594.

[17] H. Gustafsson et al. Phys. Rev. Lett. 52 (1984) 1590

[18] T.C. Awes et al., WA 80 Collaboration, Phys. Lett. B381(1996) 29

[19] H.Heiselberg, private communication

[20] P. Huovinen, arXiv:nucl-th/0210024

[21] J.Y. S. Voloshin and Y.Zhang, Z.Phys.C70,665(1996); A

[22] Ollitrault, Nucl. Phys. A 638(1998)195c-206c sis 